\title{
ASYMPTOTIC BEHAVIOUR OF COMPOUND DISTRIBUTIONS
}

\author{
By Paul Embrechts* \\ Imperial College, London, U.K. \\ Makoto Maejima \\ Keio University, Yokohama, Japan \\ AND \\ JozeF L. Teugels \\ Departement Wiskunde, KUL, Leuven, Belgium
}

Abstract

We improve on some results of SUNDT (1982) on the asymptotic behaviour of compound negative binomial distributions.

KEY WORDS

Compound negative binomial distributions, renewal theory, asymptotic estimates.

Consider the aggregate claims of an insurance company in a given period,

$$
X=\sum_{i=1}^{N} Y_{i}
$$

where the claim sizes $\left\{Y_{i}: i \in \mathbb{N}_{0}\right\}$ are i.i.d. non-negative random variables with $F(x)=P\left\{Y_{1} \leqslant x\right\}$ non-lattice (i.e., we assume the claim size distribution $F$ to be non-discrete; take for instance $F$ continuous), independent of the negative binomial claim arrival variable $N$. Then

$$
p_{n} \equiv P\{N=n\}=\left(\begin{array}{c}
\alpha-1+n \\
n
\end{array}\right) p^{n} q^{\alpha}, \quad n \in \mathbb{N}
$$

where $0<p<1, p+q=1$ and $\alpha>0$. Denote by $f$ the Laplace-Stieltjes transform of $F$ and assume that there exists a constant $\kappa>0$ satisfying

$$
p^{-1}=\int_{0}^{\infty} e^{\kappa x} d F(x)
$$

and that

$$
\nu=p \int_{0}^{\infty} x e^{\kappa x} d F(x)<\infty
$$

i.e., $p^{-1}=f(-\kappa)$ and $\left|f^{\prime}(-\kappa)\right|<\infty$. We now want to estimate $P\{X>x\}$ as $x \rightarrow \infty$.

* This research was supported by a grant from The Nuffield Foundation.

ASTIN BULLETIN Vol. 15, No. 1 
Notation. If $f(n)$ and $g(n)$ are two functions, in this paper we always abbreviate the statement $\lim _{n \rightarrow \infty} f(n) / g(n)=1$ to $f(n) \sim g(n)$ as $n \rightarrow \infty$.

In SUNDT (1982), the following theorem was proved.

Proposition 1 (Sundt (1982), Theorem 5). If $e^{\kappa x} P\{X>x\}$ is ultimately monotone, then

$$
P\{X>x\} \sim(\kappa \Gamma(\alpha))^{-1}(q / \nu)^{\alpha} x^{\alpha-1} e^{-\kappa x}, \text { as } x \rightarrow \infty .
$$

The condition of ultimate monotonicity was needed because the proof in SUNDT (1982) used a Tauberian argument. In this note we want to prove that under (1) and (2), (3) always holds, as indeed was conjectured by Sundt.

THEOREM. Assume the negative binomial model above. If the claim size distribution $F$ satisfies (1) and (2), then (3) holds.

Of course condition (2) is only needed to get a non-trivial statement in (3). The proof of the theorem differs entirely from the one given in SUNDT (1982) and essentially hinges on the following recent Blackwell type theorem for generalised renewal measures.

Proposition 2 (Embrechts, Maejima And Omey (1984), Theorem 1a). Let $a$ be a positive function such that $a(x)=x^{\beta} L(x), \beta>-1$ and $L$ slowly varying (that is, for all $t>0, L(t x) \sim L(x)$ as $x \rightarrow \infty)$. Let $F$ be non-lattice. Then for all $h>0$,

$$
\sum_{n=1}^{\infty} a(n) P\left\{x<S_{n} \leqslant x+h\right\} \sim h \mu^{-\beta-1} a(x), \quad \text { as } x \rightarrow \infty,
$$

where $S_{n}=X_{1}+\cdots+X_{n}$ is the random walk defined by $F$ and $\mu$ the mean of $F$. Moreover, the convergence in (4) is uniform in $h$ on compact sets.

A more general statement including $\beta \leqslant-1$ is given in EMBRECHTS, MAEJIMA and OMEY (1984).

Proof of Theorem. Define the associated distribution or Esscher transform

$$
F_{\kappa}(x)=(f(-\kappa))^{-1} \int_{0}^{x} e^{\kappa y} d F(y), \quad x \geqslant 0 .
$$

One easily verifies that for all $n \geqslant 2$, integer, because of (1)

$$
F_{\kappa}^{(n)}(x)=p^{n} \int_{0}^{x} e^{\kappa y} d F^{(n)}(y), \quad x \geqslant 0
$$

(here $(n)$ denotes the $n$th convolution, i.e., $G^{(n)} \equiv G^{* n}$ ).

Now

$$
P\{X>x\}=\int_{x}^{\infty} e^{-\kappa y} d\left\{\sum_{n=0}^{\infty} p^{-n} p_{n} F_{\kappa}^{(n)}(y)\right\}
$$


By Stirling's formula, $a(n)=p^{-n} p_{n} \sim\left(q^{\alpha} / \Gamma(\alpha)\right) n^{\alpha-1}$ as $n \rightarrow \infty$, satisfying the condition in proposition 2 with $\beta=\alpha-1$. Hence it follows that, with

$$
\begin{gathered}
H(y)=\sum_{n=0}^{\infty} p^{-n} p_{n} F_{\kappa}^{(n)}(y), \quad y \geqslant 0, \\
\forall h>0: H(y+h)-H(y) \sim h \nu^{-\alpha} a(y), \quad y \rightarrow \infty .
\end{gathered}
$$

In this last expression, we use $a(\cdot)$ defined on the positive real numbers, this can be achieved most easily by $a(x)=a([x])$ where [] denotes integer part. Therefore by uniform convergence:

$$
\forall \varepsilon>0 \exists y^{*}: \forall y \geqslant y^{*} \text { and } \forall h, 0 \leqslant h \leqslant 1 \text {, say: }
$$

$$
\frac{1-\varepsilon}{\Gamma(\alpha)}(q / \nu)^{\alpha} h y^{\alpha-1} \leqslant H(y+h)-H(y) \leqslant \frac{1+\varepsilon}{\Gamma(\alpha)}(q / \nu)^{\alpha} h y^{\alpha-1} \text {. }
$$

Take now $x \geqslant y=y^{*}(\varepsilon), \Delta>0$ fixed then it follows from (5) and (6) that

$$
\begin{aligned}
P\{X>x\} & =\int_{x}^{\infty} e^{-\kappa y} d H(y) \\
& =\sum_{k=0}^{\infty} \int_{x+k \Delta}^{x+(k+1) \Delta} e^{-\kappa y} d H(y) \\
& \leqslant \sum_{k=0}^{\infty} e^{-\kappa(x+k \Delta)}(H(x+(k+1) \Delta)-H(x+k \Delta)) \\
& \leqslant \frac{1+\varepsilon}{\Gamma(\alpha)}(q / \nu)^{\alpha} \sum_{k=0}^{\infty} e^{-\kappa(x+k \Delta)}(x+k \Delta)^{\alpha-1} \Delta \\
& \rightarrow \frac{1+\varepsilon}{\Gamma(\alpha)}(q / \nu)^{\alpha} \int_{x}^{\infty} e^{-\kappa y} y^{\alpha-1} d y, \text { as } \Delta \downarrow 0 .
\end{aligned}
$$

A similar argument, replacing $(1+\varepsilon)$ by $(1-\varepsilon)$, proves the converse inequality $(\geqslant)$. Letting $\varepsilon \downarrow 0$ we get as $x \rightarrow \infty$

$$
P\{X>x\} \sim \frac{1}{\Gamma(\alpha)}(q / \nu)^{\alpha} \int_{x}^{\infty} e^{-\kappa y} y^{\alpha-1} d y .
$$

The theorem follows since

$$
\int_{x}^{\infty} e^{-\kappa y} y^{\alpha-1} d y \sim \kappa^{-1} e^{-\kappa x} x^{\alpha-1}, \text { as } x \rightarrow \infty .
$$

Obviously, there is no mistery in assuming $p_{n}$ to be negative binomial. The proof easily extends to more general situations. To give the reader some idea of the generality of our approach, below we present a fairly straightforward extension of our Theorem. For further details, the reader is referred to Teugels (1985) in which these and related questions in insurance will be discussed.

For instance, suppose $p_{n}=P\{N=n\}$ satisfies the following property: there exist $\mu>1, L$ slowly varying and $\gamma \in \mathbb{R}$ such that $\mu^{n} p_{n} \sim n^{\gamma} L(n)$ as $n \rightarrow \infty$ (in the 
negative binomial case, $\mu=p^{-1}, L(n) \equiv\left(q^{\alpha} / \Gamma(\alpha)\right)$ and $\left.\gamma=\alpha-1\right)$. Again, define the Esscher transform (assumed to exist!) by

$$
F_{\kappa}(x)=(f(-\kappa))^{-1} \int_{0}^{x} e^{\kappa y} d F(y)
$$

where $\kappa=\kappa(\mu)>0$ is the solution of

$$
\mu=\int_{0}^{\infty} e^{\kappa x} d F(x)
$$

If now the conditions (1) and (2) hold (with $p^{-1}$ replaced by $\mu$ ) then

$$
P\{X>x\} \sim \frac{\left(-f^{\prime}(-\kappa) / f(-\kappa)\right)^{-\gamma-1}}{\kappa} e^{-\kappa x} x^{\gamma} L(x), \quad \text { as } x \rightarrow \infty .
$$

In general, the behaviour of $P\{X>x\}$ will depend on the relationship between the asymptotic behaviour of $P\{N>n\}$ and $1-F(x)$ as $n, x \rightarrow \infty$. A multitude of results exist, these are all reviewed in TEUGELs (1985). In a forthcoming paper, we also plan to return to the lattice case (i.e., when the claim size distribution is discrete).

\section{REFERENCES}

Embrechts, P., Maejima, M. and OMey, E. (1984) A Renewal Theorem of Blackwell Type. Annals of Probability 12, 561-570.

Sundt, B. (1982) Asymptotic Behaviour of Compound Distributions and Stop-Loss Premiums. Astin Bulletin 13, 89-98.

Teugels, J.L. (1985) Selected Topics in Insurance Mathematics. Lecture Notes: Catholic University of Leuven (Belgium), Department of Mathematics, to appear. 